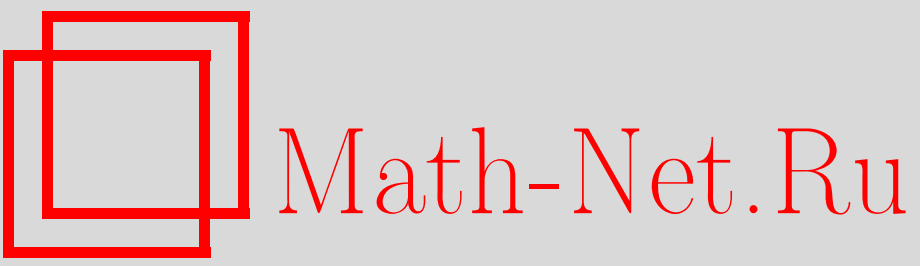

В. Ю. Королев, И. Г. Шевцова, Новая моментная оценка скорости сходимости в теореме Ляпунова, Теория вероятн. и ее примен., 2010, том 55, выпуск 3, 577582

DOI: https://doi.org/10.4213/tvp4243

Использование Общероссийского математического портала Math-Net.Ru подразумевает, что вы прочитали и согласны с пользовательским соглашением http://www.mathnet.ru/rus/agreement

Параметры загрузки:

IP : 3.85 .183 .62

26 апреля 2023 г., $15: 55: 46$ 
5. Busbridge I. W. The Mathematics of Radiative Transfer. Cambridge: Cambridge Univ. Press, 1960, 143 p. (Cambridge Tracts in Math. Phys., v. 50.)

6. Черчиньяни K. Теория и приложения уравнения Больцмана. М.: Мир, 1978, 496 с.

7. Арабаджян Л. Г., Енгибарян Н.Б. Уравнения в свертках и нелинейные функциональные уравнения. - Итоги науки и техники. Матем. анализ, т. 22. М.: ВИНИТИ, 1984, с. 175-244.

8. Енгибарян Н. Б., Мелконян Э.А. О методе дискретных ординат. - Докл. АН CCCP, 1987, т. 292, № 2, c. 322-326.

9. Yengibarian N. B. Renewal equation on the whole line. - Stochastic Process. Appl., 2000 , v. 85 , p. $237-247$.

10. Енгибарян Н. Б. Уравнения восстановления на полуоси. - Изв. РАН, сер. матем., 1999 , т. 63 , № 1, c. 61-76.

Поступила в редакцию 7.III. 2008

\section{НОВАЯ МОМЕНТНАЯ ОЦЕНКА СКОРОСТИ СХОДИМОСТИ В TЕОРЕМЕ ЛЯПУНОВА ${ }^{1)}$}

Для равномерного расстояния $\Delta_{n}$ между функцией распределения стандартного нормального закона и функцией распределения нормированной суммы произвольного числа $n \geqslant 1$ независимых случайных величин $X_{1}, \ldots, X_{n}$, имеющих нулевые средние, дисперсии $\sigma_{i}^{2}=\mathbf{D} X_{i}>0$ и третьи абсолютные моменты $\beta_{i}=\mathbf{E}\left|X_{i}\right|^{3}, i=1, \ldots, n$, доказано неравенство

$$
\Delta_{n} \leqslant 0.3197 \cdot \sum_{i=1}^{n}\left(\beta_{i}+\sigma_{i}^{3}\right)\left(\sum_{i=1}^{n} \sigma_{i}^{2}\right)^{-3 / 2} .
$$

Ключевые слова и фразы: центральная предельная теорема, неравенство Берри-Эссеена, абсолютная константа.

Пусть $X_{1}, \ldots, X_{n}$ - независимые случайные величины, заданные на некотором вероятностном пространстве $(\Omega, \mathscr{A}, \mathbf{P})$ и удовлетворяющие условиям

$$
\mathbf{E} X_{i}=0, \quad \mathbf{D} X_{i}=\sigma_{i}^{2}>0, \quad \mathbf{E}\left|X_{i}\right|^{3}=\beta_{i}<\infty, \quad i=1, \ldots, n .
$$

Обозначим $S_{n}=\left(X_{1}+\cdots+X_{n}\right) / \sqrt{\sigma_{1}^{2}+\cdots+\sigma_{n}^{2}}$. Функцию распределения случайной величины $S_{n}$ и стандартную нормальную функцию распределения соответственно обозначим $F_{n}(x)$ и $\Phi(x)$. Для удобства введем векторные обозначения $\vec{\sigma}=\left(\sigma_{1}, \ldots, \sigma_{n}\right)$, $\vec{\beta}=\left(\beta_{1}, \ldots, \beta_{n}\right)$.

* Московский государственный университет им. М. В. Ломоносова, факультет вычислительной математики и кибернетики, Ленинские горы, 119991 Москва, Россия; Институт проблем информатики РАH; e-mail: vkorolev@cs.msu.su

** Московский государственный университет им. М. В. Ломоносова, факультет вычислительной математики и кибернетики, Ленинские горы, 119991 Москва, Россия; e-mail: ishevtsova@cs.msu.su

1) Работа выполнена при поддержке Российского фонда фундаментальных исследований (проекты 08-01-00563, 08-01-00567, 08-07-00152 и 09-07-12032-офи-м), а также Министерства образования и науки (государственные контракты П1181, П779 и П958, грант МK-581.2010.1) 
В 1941 г. Э. Берри [10] показал, что в сделанных выше предположениях

$$
\Delta_{n} \equiv \sup _{x \in \mathbf{R}}\left|F_{n}(x)-\Phi(x)\right| \leqslant C_{0} \psi_{1},
$$

где $C_{0}$ - конечная положительная абсолютная постоянная,

$$
\psi_{1}=\psi_{1}(\vec{\sigma}, \vec{\beta})=\left(\sum_{i=1}^{n} \sigma_{i}^{2}\right)^{-1 / 2} \max _{1 \leqslant i \leqslant n} \frac{\beta_{i}}{\sigma_{i}^{2}} .
$$

Независимо от Берри в 1942 г. К.-Г. Эссеен [11] доказал неравенство

$$
\Delta_{n} \leqslant C_{0} \psi_{2}
$$

где $C_{0}-$ конечная положительная абсолютная постоянная,

$$
\psi_{2}=\psi_{2}(\vec{\sigma}, \vec{\beta})=\left(\sum_{i=1}^{n} \sigma_{i}^{2}\right)^{-3 / 2} \sum_{i=1}^{n} \beta_{i} .
$$

Несложно убедиться, что $\psi_{2} \leqslant \psi_{1}$, причем в случае, когда слагаемые $X_{1}, \ldots, X_{n}$ имеют одинаковые распределения, $\psi_{2}=\psi_{1}=\beta_{1} /\left(\sigma_{1}^{3} \sqrt{n}\right)$. В связи с этими обстоятельствами неравенство (2) принято называть неравенством Берри-Эссеена. При этом относительно константы $C_{0}$ в (2) известно, что

$$
0.4097 \approx \frac{\sqrt{10}+3}{6 \sqrt{2 \pi}} \leqslant C_{0} \leqslant 0.5600 .
$$

Нижняя оценка установлена К.-Г. Эссееном в 1956 г. [12], верхняя — недавно И.Г. Шевцовой [9]. Как показано в [4], если слагаемые имеют одинаковые распределения, то $C_{0} \leqslant 0.4784$. Богатая результатами и коллизиями история уточнения верхних оценок для $C_{0}$ подробно описана в работах [2], [4], [13].

В 1966 г. была опубликована работа С. Цаля [18], в которой доказано неравенство

$$
\Delta_{n} \leqslant 0.65 \cdot \psi_{3},
$$

где

$$
\begin{gathered}
\psi_{3}=\psi_{3}(\vec{\sigma}, \vec{\beta})=\left(\sum_{i=1}^{n} \sigma_{i}^{2}\right)^{-3 / 2} \sum_{i=1}^{n} \beta_{i}^{\prime}, \\
\beta_{i}^{\prime}= \begin{cases}\beta_{i}, \quad & \beta_{i} \frac{3 \sigma_{i}^{2}}{\sqrt{2}}, \\
\frac{3 b \sigma_{i}^{3}}{\sqrt{2} b+1-\sqrt{2} \beta_{i} /\left(3 \sigma_{i}^{3}\right)}, & i=1, \ldots, n,\end{cases}
\end{gathered}
$$

$b=1.07859$. Несложно проверить, что если

$$
\sqrt{2} \beta_{i} \sigma_{i}^{-2}<3<\sqrt{2} \beta_{i} \sigma_{i}^{-3}, \quad i=1, \ldots, n,
$$

то $\beta_{i}^{\prime}<\beta_{i}, i=1, \ldots, n$, и, следовательно, в таком случае $\psi_{3}<\psi_{2}$. Как показано в [18], в общем случае $\psi_{3}(\vec{\sigma}, \vec{\beta}) \leqslant 1.58 \cdot \psi_{2}(\vec{\sigma}, \vec{\beta})$, причем максимальное значение 1.58 отношения $\psi_{3} / \psi_{2}$ достигается на симметричных бернуллиевских распределениях. Поэтому, вообще говоря, функции $\psi_{2}(\vec{\sigma}, \vec{\beta})$ и $\psi_{3}(\vec{\sigma}, \vec{\beta})$ несравнимы. На момент опубликования работы [18] наилучшей верхней оценкой константы $C_{0}$ в $(2)$ была $C_{0} \leqslant 1.98$ [17], в то время как из $(3)$ вытекает оценка $C_{0} \leqslant 0.65 \cdot 1.58=1.027$. Поэтому неравенство (3) стало определенным продвижением в задаче оценивания точности нормальной аппроксимации для распределений сумм независимых случайных величин в терминах моментов слагаемых. С другой стороны, значение работы [18], в частности, и в том, что в ней было продемонстрировано, что, выбирая форму зависимости мажоранты от моментов, отличную от простой и удобной классической функции $\psi_{2}(\vec{\sigma}, \vec{\beta})$, можно 
заметно уточнить неравенство Берри-Эссеена, что особенно важно при практических расчетах.

Здесь уместно подробнее остановиться на вопросе о том, почему в данной работе мы сосредоточились на моментных оценках. Этот вопрос тесно связан с вопросом о том, что считать оценкой. Самой точной и правильной оценкой равномерного расстояния является, очевидно, само равномерное расстояние: $\Delta_{n} \leqslant \Delta_{n}$, но по своему смыслу оценка должна иметь более простой вид по сравнению с оцениваемой величиной и эффективно вычисляться, требуя лишь некоторую наиболее доступную информацию об исходных распределениях. Конечно же, неравенства в терминах псевдомоментов (см., например, [5]) или дзета-метрик (см., например, [7]) могут быть существенно точнее оценок, рассматриваемых в данной заметке. Однако чтобы вычислить характеристики, участвующие в указанных неравенствах (псевдомоменты или дзета-метрики), необходима полная информация о распределениях слагаемых. Но при этом, естественно, имея такую информацию и современные компьютеры, на практике вполне можно оценить погрешность нормальной аппроксимации численно, не прибегая к аналитическим методам. В неравенствах же моментного типа вся необходимая информация сосредоточена лишь в простых характеристиках интегрального типа (первых трех моментах), которые, как правило, можно эффективно оценить по выборке, особенно в случае одинаково распределенных слагаемых. Какими из перечисленных методов пользоваться для оценивания точности нормальной аппроксимации на практике - дело вкуса, но при этом ценой за использование более точных оценок является доступ к более полной информации о распределениях слагаемых.

В данной работе для случая не обязательно одинаково распределенных слагаемых предлагается альтернативная классическому неравенству Берри-Эссеена моментная оценка с уточненной структурой в духе идей, реализованных ранее в наших работах [2]-[4], [13] для случая, в котором слагаемые имеют одно и то же распределение.

Для упрощения выкладок всюду далее будем считать, что $\sigma_{1}^{2}+\cdots+\sigma_{n}^{2}=1$. Обозначим

$$
\ell_{n}=\sum_{i=1}^{n} \beta_{i}, \quad \tau_{n}=\sum_{i=1}^{n} \sigma_{i}^{3}, \quad \varepsilon_{n}=\ell_{n}+\tau_{n}, \quad n=1,2, \ldots
$$

Теорема 1. Для любого $n \geqslant 1$ справедливо неравенство

$$
\Delta_{n} \leqslant C_{1} \varepsilon_{n}
$$

При этом $C_{1} \leqslant 0.3197$.

3 а м е ч а н и е 1 . Верхняя оценка константы $C_{1}$, приведенная в теореме 1 , оказывается меньше теоретически минимально возможного значения $0.4097 \ldots$ константы $C_{0}$ в $(2)$. При этом $\tau_{n} / \ell_{n} \leqslant 1$ в силу неравенства Ляпунова. Если $\tau_{n} / \ell_{n} \leqslant 0.75$, то неравенство (4) с $C_{1} \leqslant 0.3197$ устанавливает более точную оценку равномерного расстояния $\Delta_{n}$, чем неравенство Берри-Эссеена $(2)$ с наилучшей известной верхней оценкой константы $C_{0}=0.5600 ;$ если же $\tau_{n} / \ell_{n} \leqslant 0.28$, то (4) точнее, чем (2) даже с теоретически минимально возможным значением константы $C_{0}=0.4097 \ldots$.

3 а м е ч а н и е 2 . Если слагаемые $X_{1}, X_{2}, \ldots$ имеют одинаковые распределения, то, как показано в работах [4] и [17], $C_{1} \leqslant 0.3041$.

3 а м е ч а н и е 3 . Для $C_{1}$ справедлива нижняя оценка $C_{1} \geqslant 2 /(3 \sqrt{2 \pi})=$ $0.2659 \ldots$ (см. [3], [8]).

Доказательство теоремы 1 основано на методе В. М. Золотарева, усовершенствованном при помощи неравенства сглаживания Х. Правитца [14] и некоторых оценок для характеристических функций [4], [7]. Ниже приведем лишь основные идеи, формулируя соответствующие утверждения в виде лемм. 
Обозначим

$$
f(t)=\mathbf{E} e^{i t S_{n}}=\prod_{j=1}^{n} f_{j}(t), \quad f_{j}(t)=\mathbf{E} e^{i t X_{j}}, \quad r(t)=\left|f(t)-e^{-t^{2} / 2}\right|, \quad t \in \mathbf{R} .
$$

Лемма 1 (см. [14]). Для всех $t_{0} \in(0,1]$ u $T>0$ справедлива оченка

$$
\begin{aligned}
\Delta_{n} \leqslant & 2 \int_{0}^{t_{0}}|K(t)| r(T t) d t+2 \int_{t_{0}}^{1}|K(t)| \cdot|f(T t)| d t \\
& +2 \int_{0}^{t_{0}}\left|K(t)-\frac{i}{2 \pi t}\right| e^{-T^{2} t^{2} / 2} d t+\frac{1}{\pi} \int_{t_{0}}^{\infty} e^{-T^{2} t^{2} / 2} \frac{d t}{t},
\end{aligned}
$$

где

$$
K(t)=\frac{1}{2}(1-|t|)+\frac{i}{2}\left[(1-|t|) \operatorname{ctg} \pi t+\frac{\operatorname{sign} t}{\pi}\right], \quad-1 \leqslant t \leqslant 1 .
$$

Оценки для характеристических функций устанавливаются следующими леммами. Пусть $\theta_{0} \approx 3.995895$ - единственный корень уравнения

$$
\begin{gathered}
\theta^{2}+2 \theta \sin \theta+6(\cos \theta-1)=0, \quad \pi \leqslant \theta \leqslant 2 \pi, \\
\varkappa \equiv \sup _{x>0} \frac{\left|\cos x-1+x^{2} / 2\right|}{x^{3}} \approx 0.09916 .
\end{gathered}
$$

Можно убедиться, что супремум достигается в точке $x=\theta_{0}$. Для $t \in \mathbf{R}$ и $\varepsilon>0$ введем функцию

$$
\Psi(t, \varepsilon)= \begin{cases}t^{2} / 2-\varkappa \varepsilon|t|^{3}, & \varepsilon|t|<\theta_{0}, \\ \frac{1-\cos (\varepsilon t)}{\varepsilon^{2}}, & \theta_{0} \leqslant \varepsilon|t| \leqslant 2 \pi, \\ 0, & \varepsilon|t|>2 \pi .\end{cases}
$$

Несложно убедиться, что функция $\Psi(t, \varepsilon)$ монотонно убывает по $\varepsilon$ при каждом фиксированном $t \in \mathbf{R}$.

Лемма 2 (см. [15]). Для любого $t \in \mathbf{R}$ справедливо неравенство $|f(t)| \leqslant$ $\exp \left\{-\Psi\left(t, \varepsilon_{n}\right)\right\}$.

Лемма 3. Для любого $t \in \mathbf{R}$

$$
r(t) \leqslant 2 e^{-t^{2} / 2} \int_{0}^{|t|} \sin \left(\frac{u \varepsilon_{n}}{4} \wedge \frac{\pi}{2}\right) u \exp \left\{2 \varkappa \ell_{n} u^{3}+g\left(\left(\frac{\varepsilon_{n}}{2}\right)^{1 / 3} u \wedge \frac{1}{6 \varkappa}\right)\right\} d u
$$

где $g(u)=u^{2} / 2-2 \varkappa u^{3}, u \geqslant 0$, причем $g(u)$ монотонно возрастает при $0 \leqslant u \leqslant$ $(6 \varkappa)^{-1}$.

Данная лемма является следствием оценок, полученных в работах [4], [6], и замечания о том, что $\ell_{n} \leqslant \varepsilon_{n}$,

$$
\sigma_{k} \leqslant\left(\frac{\beta_{k}+\sigma_{k}^{3}}{2}\right)^{1 / 3} \leqslant\left(\frac{\varepsilon_{n}}{2}\right)^{1 / 3}, \quad 0 \leqslant k \leqslant n .
$$

Лемма 4 (см. [1]). Для любой функиии распределения $F$ с нулевьм средним и единичной дисперсией

$$
\sup _{x \in \mathbf{R}}|F(x)-\Phi(x)| \leqslant \sup _{x>0}\left(\Phi(x)-\frac{x^{2}}{1+x^{2}}\right)=0.54093 \ldots
$$
1.692 .

Лемма 4 позволяет не рассматривать область значений $\varepsilon_{n} \geqslant 0.54094 / 0.3197 \approx$ 
Лемма 5 (см. [16]). При условии $\left(1-\max _{1 \leqslant k \leqslant n} \sigma_{k}^{2}\right)^{-3 / 2} \varepsilon_{n} \leqslant 0.2$ справедлива оченка

$$
\Delta_{n} \leqslant\left(0.2731 \ell_{n}+0.2420 \tau_{n}\right)\left(1-\max _{1 \leqslant k \leqslant n} \sigma_{k}^{2}\right)^{-3 / 2} .
$$

Поскольку $\sup _{1 \leqslant k \leqslant n} \sigma_{k}^{2} \leqslant\left(\varepsilon_{n} / 2\right)^{2 / 3}$, при $\varepsilon_{n} \leqslant 0.06$ из леммы 5 получаем оценку $\Delta_{n} \leqslant 0.3181 \cdot \varepsilon_{n}$, что позволяет при доказательстве теоремы 1 не рассматривать область $\varepsilon_{n} \leqslant 0.06$.

Подставляя оценки для $|f(t)|$ и $r(t)$ из лемм 2 и 3 в правую часть неравенства сглаживания Правитца из леммы 1 , получаем некоторую функцию $D\left(\varepsilon_{n}, t_{0}, T\right)$, мажорирующую равномерное расстояние $\Delta_{n}$ при всех $t_{0} \in(0,1], T>0$ и $\varepsilon_{n}>0$. Таким образом, константу $C_{1}$ оказывается возможным искать в виде

$$
C_{1}=\max _{0.06 \leqslant \varepsilon \leqslant 1.7} C(\varepsilon), \quad C(\varepsilon)=\inf _{t_{0}, T} \frac{D\left(\varepsilon, t_{0}, T\right)}{\varepsilon} .
$$

Основываясь на монотонности используемых мажорант для $|f(t)|$ и $r(t)$ по $\varepsilon_{n}$, заключаем что функция $\varepsilon C(\varepsilon)$ монотонно не убывает, а это позволяет записать неравенство

$$
C(\varepsilon) \leqslant \frac{C\left(\varepsilon_{2}\right) \varepsilon_{2}}{\varepsilon_{1}}, \quad \varepsilon_{1} \leqslant \varepsilon \leqslant \varepsilon_{2},
$$

и оценить $\sup C(\varepsilon)$ по значениям $\varepsilon$ лишь в конечном числе точек.

Bce необходимые вычисления были произведены в среде Matlab R2006b. Оказалось, что экстремальное значение 0.31965 функции $C(\varepsilon)$ доставляет $\varepsilon \approx 0.964$ $\left(t_{0} \approx 0.3047, T \approx 6.2730\right)$, что и доказывает теорему.

\section{СПИСОК ЛИТЕРАТУРЫ}

1. Бхаттачария Р. Н., Ранга Рао Р. Аппроксимация нормальным распределением. М.: Наука, 1982, 286 с.

2. Королев В.Ю., Шевцова И. Г. О верхней оценке абсолютной постоянной в неравенстве Берри-Эссеена. - Теория вероятн. и ее примен., 2009, т. 54, в. 4, с. 671695.

3. Королев В. Ю., Шевцова И. Г. Уточнение неравенства Берри-Эссеена. - Докл. PAH, 2010, т. 430, № 6, с. 738-742.

4. Королев В.Ю., Шевцова И. Г. Уточнение неравенства Берри-Эссеена с приложениями к пуассоновским и смешанным пуассоновским случайным суммам. Обозрение прикл. и промышл. матем., 2010, т. 17 , в. 1, с. 25-56.

5. Паулаускас В.И. Об одном усилении теоремы Ляпунова. - Литов. матем. сб., 1969, т. 9, № 2, с. 173-179.

6. Тюрин И. С. О точности гауссовской аппроксимации. - Докл. РАН, 2009, т. 429, № 3, с. 312-316.

7. Тюрин И. С. Уточнение верхних оценок констант в теореме Ляпунова. - Успехи матем. наук, 2010, т. 65, в. 3(393), с. 201-202.

8. Шевцова И. Г. Нижняя асимптотически правильная постоянная в центральной предельной теореме. - Докл. РАН, 2010, т. 430, № 4, с. 466-469.

9. Шевцова И.Г. Уточнение оценок скорости сходимости в теореме Ляпунова. Докл. РАН, 2010 (в печати).

10. Berry A.C. The accuracy of the Gaussian approximation to the sum of independent variables. - Trans. Amer. Math. Soc., 1941, v. 49. p. 122-136.

11. Esseen C.-G. On the Liapunoff limit of error in the theory of probability. Ark. Mat. Astron. Fys., 1942, v. A28, № 9, p. 1-19; http://www.infrmaworld.com/ 10.1080/03461238.2010.485370.

12. Esseen C.-G. A moment inequality with an application to the central limit theorem. Skand. Aktuarietidskr., 1956, v. 39, p. 160-170. 
13. Korolev V. Yu., Shevtsova I. G. An improvement of the Berry-Esseen inequality with applications to Poisson and mixed Poisson random sums. - Scand. Actuar. J., 2010 (to appear).

14. Prawitz $H$. Limits for a distribution, if the characteristic function is given in a finite domain. - Scand. Aktuarietidskr., 1972, № 2, P. 138-154.

15. Prawitz $H$. Weitere Ungleichungen für den absoluten Betrag einer charakteristischen Funktion. - Scand. Actuar. J., 1975, № 1, p. 21-28.

16. Prawitz H. On the remainder in the central limit theorem. - Scand. Actuar. J., 1975, № 3, p. $145-156$.

17. Wallace D. L. A corrected computation of Berry's bound for the central limit theorem error. - Chicago: Stat. Res. Cent., University of Chicago, 1959.

18. Zahl S. Bounds for the central limit theorem error. - SIAM J. Appl. Math., 1966, v. 14, № 6, p. 1225-1245.

Поступила в редакцию

1.VI.2010

(c) 2010 г.

МУМИнов М. C.*

\title{
О ПРЕДЕЛЬНОМ РАСПРЕДЕЛЕНИИ МАКСИМАЛЬНОГО УКЛОНЕНИЯ ЭМПИРИЧЕСКОЙ ПЛОТНОСТИ РАСПРЕДЕЛЕНИЯ И ФУНКЦИИ РЕГРЕССИИ. І.
}

\begin{abstract}
В работе для неизвестной плотности распределения $f(t), t \in \mathbf{R}^{\nu}$, случайного вектора $X \in \mathbf{R}^{\nu}$ и функции регрессии $r(t)=\mathbf{E}(Y \mid X=t)$ случайного вектора $(X, Y), X \in \mathbf{R}^{\nu}, Y \in \mathbf{R}^{1}$, построены непараметрические ядерные оценки $f_{n}(t)$ и $r_{n}(t)$ соответственно. Доказано, что распределение максимального уклонения этих оценок от истинных плотности распределения $f(t)$ и функции регрессии $r(t)$ стремится к двойному экспоненциальному закону при $n \rightarrow \infty$. С помощью построенных оценок найдена доверительная область для $f(t)$ и $r(t)$, отвечающая заданному коэффициенту доверия $\alpha(0<\alpha<1)$, и построен критерий для проверки гипотезы $H_{0}: f(t)=f_{0}(t)$ (соответственно $\left.H_{0}^{\prime}: r(t)=r_{0}(t)\right)$, где $f_{0}(t)-$ заранее заданная плотность распределения вероятностей, $r_{0}(t)$ - некоторая заданная функция.
\end{abstract}

Ключевые слова и фразы: последовательность случайных векторов, плотность распределения вероятностей, функция регрессии, непараметрические ядерные оценки, винеровский процесс с многомерным параметром, локальная стационарность случайных полей, доверительная область, проверка статистических гипотез.

1. Введение. Пусть $\nu$-мерный случайный вектор $X$ имеет неизвестную плотность распределения вероятностей $f(t), t \in \mathbf{R}^{\nu}$, а $(\nu+1)$-мерный случайный вектор $(X, Y), X \in \mathbf{R}^{\nu}, Y \in \mathbf{R}^{1}$, имеет неизвестную функцию регресии $r(t)=\mathbf{E}(Y \mid X=t)$. Пусть далее $X_{1}, \ldots, X_{n}$ и $\left(X_{1}, Y_{1}\right), \ldots,\left(X_{n}, Y_{n}\right)$ - простые выборки объема $n$ из генеральных совокупностей $X$ и $(X, Y)$ соответственно.

В качестве приближений для неизвестных плотности распределения $f(t)$ и функции регрессии $r(t)$ примем статистики

$$
f_{n}(t)=\frac{1}{n} \sum_{i=1}^{n} K_{n}\left(t, X_{i}\right),
$$

* Институт математики и информационных технологий АН РУз, отдел теории вероятностей и математической статистики, ул. Ф. Ходжаева, 29, 100125, Ташкент, Узбекистан; e-mail: m.muhammad@rambler.ru 